\title{
La technique du « point d'Argentan » dévoilée
}

The technique of « point d'Argentan » unveiled

\section{Brigitte Tambrun}

\section{(2) OpenEdition}

\section{Journals}

Édition électronique

URL : http://journals.openedition.org/artefact/4898

DOI : 10.4000/artefact.4898

ISSN : 2606-9245

\section{Éditeur :}

Association Artefact. Techniques histoire et sciences humaines, Presses universitaires du Midi

\section{Édition imprimée}

Date de publication : 15 décembre 2019

Pagination : 279-307

ISBN : 978-2-8107-0667-9

ISSN : 2273-0753

Référence électronique

Brigitte Tambrun, «La technique du « point d'Argentan » dévoilée », Artefact [En ligne], 11 | 2019, mis en ligne le 27 novembre 2020, consulté le 29 novembre 2020. URL : http://journals.openedition.org/ artefact/4898; DOI : https://doi.org/10.4000/artefact.4898

\section{$\Theta \oplus \Theta \Theta$}

Artefact, Techniques, histoire et sciences humaines est mise à disposition selon les termes de la Licence Creative Commons Attribution - Pas d'Utilisation Commerciale - Pas de Modification 4.0 International. 


\section{La technique du «point d’Argentan » dévoilée}

\section{Brigitte Tambrun}

\section{Résumé}

Comment une technique de dentelle à l'aiguille qui était pratiquée au xvIII siècle par des femmes, et par des enfants dès l'âge de six ans, dans les manufactures d'Alençon et d'Argentan, est-elle devenue au $x x^{\mathrm{e}}$ siècle un secret bien gardé au sein du monastère bénédictin d'Argentan ? Dans cet article nous montrerons que le « grand réseau d'Argentan » des ateliers de la maison Lefébure, reconstitué à Bayeux vers 1874, correspond à ce que l'on appelait au xvIII siècle un " champ de bride ». Or la technique d'exécution de ce « champ » est en fait minutieusement décrite dans une célèbre encyclopédie du xvIII siècle, celle de Panckoucke ; avant la Révolution française, ce point n'était donc pas tenu secret. L'Encyclopédie méthodique livre également des renseignements précieux sur les conditions sociales du travail du " Point » à l'aiguille, en ville et à la campagne; nous les mettrons en perspective avec celles du xix siècle.

\section{Mots-clés}

Argentan, Alençon, Bayeux, dentelle à l'aiguille, Point de France, Point d'Argentan, Point d'Alençon, champ de bride, bride tortillée, bride bouclée, bride épinglée, Lefébure

99 Brigitte Tambrun, « La technique du "point d’Argentan" dévoilée », Artefact, 11, 2019, p. 279-307. 


\section{The technique of " point d'Argentan " unveiled}

\section{Abstract}

How is it that a technique of needle lace-making, which was practiced in the eighteenth century by women-and by children from the age of six on-in the workshops of Alençon and Argentan (both towns on Normandy) came to be a well-guarded secret in a Benedictine monastery in Argentan in the twentieth century? This article seeks to show how the "grand réseau of Argentan" of the workshops of the Maison Lefébure, reassembled at Bayeux around 1874, corresponds to what was called in the eighteenth century the "champ de bride." The particular technique for the production of this champ was in effect carefully described in the famous Encyclopédie Méthodique of Charles-Joseph Panckoucke in the eighteenth century, and thus before the French Revolution this lace-making technique was not kept secret. The Encyclopédie Méthodique also offers invaluable information on the social conditions of the production of needle lace-making in towns and in the countryside. The article puts these in perspective in relation to those of the nineteenth century.

\section{Keywords}

Argentan, Alençon, Bayeux, needle lace, Point de France, Argentan lace, Alençon lace, twisted bride, Lefébure

\section{Note de l'autrice}

L'article est écrit avec la collaboration de Véronique Thomazo, dentellière à l'aiguille, diplômée, enseignante à la Maison des dentelles d'Argentan, puis au Conservatoire de la dentelle de Bayeux. 


\section{Le « Point d'Argentan » : technique et histoire}

Comment une technique de dentelle à l'aiguille qui était pratiquée au $\mathrm{XVIII}^{\mathrm{e}}$ siècle par des femmes et par des enfants, dès l'âge de six ans, pour le compte de manufactures et dans des hôpitaux généraux, est-elle devenue au $\mathrm{Xx}^{\mathrm{e}}$ siècle un secret bien gardé au sein du monastère bénédictin d'Argentan ? Le " grand réseau d'Argentan ", voire l'« Argentan ", des ateliers de la maison Lefébure, élaboré à Bayeux à partir de 1874 à la demande du sous-préfet et du maire d'Argentan, correspond à ce que l'on appelait au XVIII ${ }^{\mathrm{e}}$ siècle un "champ de bride ". Or nous allons montrer que la technique d'exécution de ce " champ " était minutieusement décrite dans une célèbre encyclopédie du XviII ${ }^{\mathrm{e}}$ siècle, celle de Panckoucke. Avant la Révolution française, ce point n'était pas tenu secret.

Nous nous demanderons sur quoi la distinction entre « Point d'Argentan» et «Point d'Alençon " est fondée ; si la technique du " champ de bride » qui caractériserait le "Point d'Argentan " est la même au XIx ${ }^{\mathrm{e}}$ siècle qu'au $\mathrm{XVIII}^{\mathrm{e}}$ siècle ; si les conditions sociales de la fabrication et de la commercialisation du point à l'aiguille ont changé entre l'Ancien Régime et le Second Empire.

\section{Le vocabulaire et l'histoire du « Point d'Argentan "}

Tandis que la " dentelle " proprement dite est faite à l'aide de multiples fuseaux, qui permettent de croiser et tourner de nombreux fils (il s'agit d'une forme de passement voire de tissage), le " Point ", que l'on appelle de nos jours la "dentelle à l'aiguille ", s'exécute avec une aiguillée de fil et s'apparente à la broderie. Le " Point » a pour support provisoire un morceau de vélin (parchemin de veau), doublé d'une toile pliée en deux, sur lequel on fixe la " trace ", fil double qui délimite les motifs et marque le bord de la pièce. Le " Point» se compose de motifs, qui se détachent sur un " champ ». Le « champ " figure l'arrière-plan dans une pièce de dentelle.

Au XVIII ${ }^{\mathrm{e}}$ siècle, on distingue deux types de champs : le champ de réseau (à petites mailles rectangulaires non festonnées) et le champ de bride (à 
mailles hexagonales, festonnées ou surjetées). À Argentan, la manière de faire le " champ de bride » et même le "Point » est tombée dans l'oubli après la Révolution ${ }^{1}$. C'est la maison Lefébure qui l'a finalement reconstituée en 1874.

Gérard Kempf a montré que la ville d'Argentan comptait au XvIII ${ }^{\mathrm{e}}$ siècle plusieurs manufactures de "Point " ou plutôt de "Point de France ". Quatre d'entre elles obtinrent un privilège royal ; elles fournissaient la cour en "Point royal de France $»^{2}$. Il existait aussi plusieurs manufactures de " Point » sans privilège royal. Même l'hôpital général Saint-Louis comportait une fabrique de "Point de France $»^{3}$.

La restauration du " Point » à Argentan au XIX siècle passe par la ville de Bayeux. En 1829, Auguste Lefébure (1798-1869), alors employé à Paris chez un marchand de dentelles, acquiert une fabrique établie à Bayeux, celle de Madame Carpentier-Delamare ; puis il fonde une maison de vente de dentelles (aux fuseaux) à Paris ${ }^{4}$. Entre 1830 et 1840, Auguste Lefébure transforme l'industrie dentellière de Bayeux en y introduisant la fabrication de la dentelle de soie noire de type Chantilly qu'il porte à l'excellence. À partir de 1855 il s'intéresse aussi à la "dentelle à l'aiguilles ", et tout d'abord au "Point d'Alençon »: "Une branche de la dentelle française, le Point d'Alençon, tout en vivant sur son antique réputation, restait très stationnaire. M. Lefébure avait cherché plusieurs fois à obtenir des facteurs ${ }^{6}$ d'Alençon, qu'on essayât de refaire pour lui quelques-uns des anciens points de France, avec fleurs à reliefs et fonds picotés, si appréciés sous Louis XIV et Louis XV ». Mais « les Alençonnais ne voulaient pas

\footnotetext{
1. En juin 1868, Philippe de Chennevières écrivait à propos d'une bride à trois ou quatre picots qui était selon lui une spécialité dans le Point d'Argentan : «La manière de faire la bride picotée est entièrement perdue. Des tentatives furent faites pour reprendre cette fabrication, il y a une quinzaine d'années, et on trouva une vieille ouvrière qui en avait fait dans sa jeunesse ; mais il lui fut impossible de retrouver le Point dans sa mémoire, et le projet fut abandonné " (De Chennevières, 1869, p. 16).

2. Sur ces manufactures, voir l'étude très documentée de Gérard Kempf, qui en donne la liste (Kempf, 1997 et 2010).

3. Kempf, 1997, p. 10-22 et 23.

4. Lefébure, 1913, p. 27.

5. Ibid., p. 6.

6. Les facteurs, et factrices, sont littéralement les personnes qui font faire la dentelle : elles fournissent la matière d'œuvre à l'ouvrière, récupèrent la pièce et paient l'exécutante ; ce sont des intermédiaires entre les marchands fabricants et les ouvrières : voir Poupet, 1913, p. 74-75.

7. Lefébure, 1913, p. 33.
} 
démordre du petit réseau, auquel ils étaient habitués : ils en avaient des commandes et ils se refusaient à étudier ces points anciens dont la technique était oubliée depuis longtemps ${ }^{8} »$.

Pour surmonter cette difficulté un " atelier spécial de recherches et d'échantillonnage en Point d'Alençon, travaillé à l'aiguille ", est établi dans la maison Lefébure de Bayeux'. Suite à l'achat d'un brevet, un atelier est ensuite fondé. On y met d'abord à l'étude, avec l'aide d'ouvrières que l'on fait venir de Caen, " la reproduction des beaux points à relief de l'ancien temps ${ }^{10}$ ": on déchiquette au préalable de vieux morceaux, pour analyser la manière dont ils ont été réalisés ${ }^{11}$.

Le travail de reconstitution des "points anciens" se poursuit avec ce qui va être désormais caractérisé comme « Point d'Argentan ». À cette époque la maison est dirigée par les frères Ernest et Anatole Lefébure, leur père Auguste Lefébure étant décédé en $1869^{12}$ : "C C'est en 1874 que, sur la demande instante de MM. Alphonse Béchard, sous-préfet d'Argentan, et Lebouc, maire de cette ville, MM. Lefébure frères entreprirent de faire revivre le Point d'Argentan dont la fabrication avait tout à fait cessé depuis le commencement du siècle ". Mais "pour retrouver le "secret du Point", si déplorablement perdu, il ne suffisait pas de posséder des spécimens variés de "Vraie Dentelle d'Argentan". Il fallait surtout avoir en main quelque indication, dessin ou canevas, permettant de découvrir les procédés jadis employés par les dentellières argentanaises ${ }^{13}$ ". "Sur le conseil de Monsieur Lefébure, de minutieuses recherches furent faites dans plusieurs maisons de la ville connues comme ayant été jadis des centres de fabrication $^{14}$ ». Cette enquête aboutit à une " heureuse découverte »: on apprit en effet «que les religieuses de l'hospice, arrivées à Argentan en 1864, avaient dû trouver, vers cette époque, en débarrassant les combles [...], certains

8. Ibid.

9. Ibid. Ces échantillons sont conservés et exposés au Musée d'Art et d'Histoire Baron Gérard (MAHB) à Bayeux.

10. L'expression "beaux points à relief " désigne des Points de Venise, qui sont particulièrement spectaculaires : ces Points sont à l'origine des Points de France. Le MAHB conserve plusieurs de ces pièces à gros relief produites par la maison Lefébure au XIx ${ }^{\mathrm{e}}$ siècle. Sur le " Point de Venise ", on pourra consulter Risselin-Steenebrugen, 1973, p. 11-12 et p. 38-50.

11. Lefébure, 1913, p. 34.

12. Ibid., p. 41.

13. Leboulanger, 1912, p. 30.

14. Ibid. 
parchemins et morceaux de dentelle que la vénérable supérieure, Sour Célestine, avait alors montrés à l'économe et aux administrateurs ${ }^{15}$ ». Et "Sœur Célestine, fort heureusement avait mis de côté les précieux parchemins [...] sur lesquels étaient dessinés, ou déjà en voie de formation, des fragments de dentelle ${ }^{16} »$. (Fig. XXII et XXIII, cahier couleur)

«Dès que M. Lefébure les vit, il s'écria : À la bonne heure !... Mais c'est un trésor que vous me présentez !... Avec ces matériaux et ces parcheminspatrons, je crois qu'il est possible de reconstituer la fabrication du "Vrai Point d'Argentan"17 ». M. Lefébure confia alors les vélins à Mademoiselle Désirée Hamel, l'une des meilleures alençonneuses ${ }^{18}$ de son atelier bayeusain. Il étudia avec elle " les mystérieux réseaux ${ }^{19}$ "; il " la chargea de retrouver la manière de refaire les grands réseaux qui caractérisent les anciennes dentelles d'Argentan ${ }^{20}$ ".

L'expression de "grands réseaux " est une nouveauté : Ernest Lefébure veut en réalité parler du " champ de bride ». Désirée Hamel a donc pour tâche d'analyser le champ de bride, à mailles hexagonales, qu'elle peut observer sur le parchemin, en le démontant même un peu. Il s'agit pour elle de comprendre comment est réalisée l'armature de fil cachée sous les points noués ${ }^{21}$ qui recouvrent entièrement les brides, de mettre au point une technique pour réaliser ce champ, et une méthode pour l'enseigner à des religieuses voire à des enfants : " $\mathrm{A}$ force de tâtonnements, de patience et de persévérance ${ }^{22}$ ", " défaisant mille fois ", et " refaisant les multiples mailles "23, Désirée Hamel, "grâce aux parchemins-patrons qu'elle avait sous les yeux ", "fut assez maîtresse de son travail " pour pouvoir " reconstituer intégralement " le point ${ }^{24}$. Elle "parvint à imiter d'abord, puis à faire véritablement le "Point d'Argentan", absolument tel qu'on le fabriquait

15. Ibid.

16. Ibid., p. 30-31.

17. Ibid., p. 31.

18. Le terme " alençonneuse " désigne une dentellière spécialisée dans le Point d'Alençon, qui s’effectue à l'aiguille.

19. Leboulanger, 1912, p. 31.

20. Plusieurs de ces pièces de dentelle du XviII ${ }^{\mathrm{e}}$ siècle, demeurées inachevées, sont actuellement conservées à la Maison des dentelles d'Argentan. Elles étaient déjà conservées au musée d'Argentan lorsque l'abbé Leboulanger écrivait son ouvrage (voir Leboulanger, 1912, p. 30).

21. Il s'agit, en termes actuels, de points de feston.

22. Leboulanger, 1912, p. 33.

23. Ibid., p. 31.

24. Ibid., p. 32. 
jadis $^{25}$ ". Car son " "Point" est absolument le même que celui d'autrefois, MM. Lefébure ont poussé l'archaïsme et le scrupule jusqu’à faire effilocher un morceau de vieille dentelle et à faire fabriquer du fil identique à l'ancien ${ }^{26} »$.

La dentellière de Bayeux est alors envoyée à Argentan :

Le 22 janvier 1874, en la fête de Saint-Vincent, patron de la ville, elle vint s'installer chez les Bénédictines d'Argentan, qui lui formèrent un atelier avec un certain nombre de leurs orphelines. Malgré les difficultés d'un début où elle devait tout tirer d'ellemême, $\mathrm{M}^{\text {Ile }}$ Hamel, encouragée et soutenue par les sacrifices que firent MM. Lefébure, parvint, en quelques années à reconstituer le travail du Point d'Argentan qu'elle enseigna aux enfants et à plusieurs religieuses de la maison ${ }^{27}$.

Comme l'a montré Gérard Kempf, l'emploi des termes d' "Alençon ", d' "Argentan " et de " Point de France ", pour désigner divers champs qui spécifieraient le genre du "Point " à l'aiguille, est une innovation ${ }^{28}$. L'abbé Leboulanger en donne la justification suivante : "Qu'on cesse donc de confondre Point, ou dentelle, avec Manufacture ${ }^{29}$ "; qu'on distingue les " Points" les uns des autres conformément à ce qui se fait pour toutes les dentelles, "par la spécialité de leur réseau [autrement dit de leur champ], auquel on ajoute le nom de la ville qui les produit ${ }^{30}$ ».

En instaurant des différences stylistiques qui n'existaient pas sous l'Ancien Régime, la maison Lefébure instaure une compétition entre les deux centres dentelliers d'Alençon - restauré sous l'Empire mais qui stagnerait et refuserait de faire des recherches sur les champs anciens - et d'Argentan - restauré par ses soins et qu'il va porter à l'excellence ${ }^{31}$. En réalité, sous l'Ancien Régime, les fabriques d'Alençon n'étaient pas spécialisées dans le

25. Ibid., p. 33 .

26. Ibid.

27. Lefébure, 1913, p. 45.

28. Voir Kempf, 1997 et Kempf, 2010, notamment p. 1-3.

29. Leboulanger, 1925, p. 16.

30. Ibid., p. 17. Ainsi, par exemple, on peut fabriquer de la "Chantilly " ou de l'« Alençon " à Bayeux, de la «Lille» au Puy, etc.

31. La maison Lefébure gagnera des prix d'excellence dans de nombreux concours internationaux : voir Lefébure, 1913, p. 38-39 et p. 52-58, ainsi que Cussy, 1855, p. 268-270. 
réseau $^{32}$ : on y réalisait de la bride comme du réseau ${ }^{33}$. Cependant, dans la première moitié du XIX ${ }^{e}$ siècle, la technique de la bride pratiquée à Alençon avait changé par rapport à celle du XVIII ${ }^{\mathrm{e}}$ siècle : en 1842, son armature était une couchure de fils tendus qui se croisaient de manière oblique, et non plus des mailles ${ }^{34}$. Son aspect en était un peu modifié.

Nous avons vu que selon l'abbé Leboulanger, il conviendrait de désigner les « Points » par la spécialité de leur champ. Or au XIx siècle, Alençon se spécialise dans le champ de réseau à mailles nues et pratique une bride qui ne serait pas authentique, au vu des échantillons du XVIII ${ }^{\mathrm{e}}$ siècle, retrouvés à Argentan. Au XIX siècle, Argentan se restaure comme centre de production de dentelle à l'aiguille avec pour spécialité l'authentique champ de bride $^{35}$. Pour bien se distinguer d'Alençon, l'abbé précise que cette bride d'Argentan est en réalité un réseau festonné : « le point d'Argentan, revenu à sa technique primitive, est constitué par un réseau renforcé d'un feston, bouclé à tous ses points de contact avec la maille ${ }^{36}$ ».

C'est pourquoi dans les dessins techniques réalisés pour des pièces de " dentelle à l'aiguille », et conservés dans les archives de la maison Lefébure au Musée d'Art et d'Histoire Baron Gérard ${ }^{37}$, le vocable d' "Argentan » désigne une pièce comportant un champ de bride traditionnel et celui $d^{\prime}$ " Alençon ", une pièce comportant un champ de réseau ; l'expression "Point de France "s'applique à une dentelle comportant un champ de bride à grandes mailles hexagonales picotées : il ne s'agit donc plus d'un terme générique désignant un point fait en France - et non dans un pays étranger - comme c'était l'usage aux XVII ${ }^{\mathrm{e}}$ et XVIII ${ }^{\mathrm{e}}$ siècles, dans le cadre du protectionnisme instauré par Colbert. Ainsi, par exemple, on trouve un

32. Comme l'attestent les documents cités par Despierres, 1886, p. 85.

33. La bride, comme le réseau, est encore attestée en 1842 dans la maison Docagne d'Alençon : voir Odolant-Desnos, 1842, p. 1.

34. Odolant-Desnos, 1842, p. 1.

35. Leboulanger, 1925, p. 18. L'abbé signale les confusions, et fustige madame Despierres qui induit les lecteurs en erreur : "Généralement, on se trompe sur la nature de la dentelle argentanaise. On a cru la caractériser par les noms de "Bride bouclée", et plus souvent encore de "Bride tortillée". Ce sont des inexactitudes. Mme Despierres elle-même, dont l'ouvrage : Histoire du Point d'Alençon, fait autorité en cette matière, n’a pas été plus heureuse. Son explication s'applique bien à certains réseaux d'imitation mais pas à notre "Point" ".

36. Leboulanger, 1925, p. 15-16.

37. Nous remercions mesdames Dominique Hérouard, directrice du MAHB, et Myriam Gouye, dentellière et régisseur des œuvres, qui nous ont permis de consulter ces documents le 17 décembre 2018. 
dessin préparatoire pour un "Rochet $\operatorname{Argentan}^{38}$ "; un autre dessin porte le titre "Argentan » avec les précisions suivantes : "Rectifications ", " $\mathrm{Ne}$ mettre du réseau fin que dans les parties bleues ${ }^{39}$; un autre est intitulé " $\operatorname{Arg}\left[\right.$ enta]n ; et, dans une "Garniture Argentan ${ }^{40}$ ", une partie est à faire " en grand réseau d'Argentan ${ }^{41}$ ». On trouve par ailleurs le dessin d'un "Volant Alençon ${ }^{42}$ ", celui d'une " $\mathrm{D}[\mathrm{ent}]$ elle Alençon ${ }^{43}$ " et celui d'une "Garniture Alençon ${ }^{44}$ ». Certaines pièces réalisées par les ateliers Lefébure associent l'"Alençon » et l'" Argentan " : ainsi, par exemple, un dessin porte le titre de "Volant Alençon - Argentan ${ }^{45}$ ». Enfin les dessins intitulés "Point de France. $1^{\text {re }}$ Rehausse ${ }^{46}$ " et "Volant Point de France ${ }^{47}$ " comportent un champ de bride à grandes mailles hexagonales ${ }^{48}$.

C'est donc par une sorte de métonymie, ou mieux de synecdoque, que l'expression de « Point d'Alençon » désigne désormais une dentelle à l'aiguille comportant un champ de réseau, fait de petites mailles rectangulaires non festonnées, celle de " Point d'Argentan », une dentelle à l'aiguille comportant un champ de bride à mailles hexagonales festonnées de petite dimension $^{49}$, et celle de " Point de France " une dentelle à l'aiguille comportant un champ de bride à mailles hexagonales plus grandes que les précédentes, festonnées et comportant des picots. Si le terme de "bride " courant au $\mathrm{XVIII}^{\mathrm{e}}$ siècle n'est jamais utilisé par Lefébure c'est aussi parce qu'il évoque

38. Musée d'Art et d'Histoire Baron Gérard (MAHB), pièce DC00218.01.

39. MAHB, DC00276.01.

40. MAHB, DC00197.01.

41. MAHB, DC00218.01.

42. MAHB, DC00237.01.

43. MAHB, DC0058.01.

44. MAHB, DC00219.01.

45. MAHB, DC00228.01. Voir aussi le «Volant en Point d'Argentan et Point d'Alençon, réalisé par l'école dentellière d'Argentan d'après un modèle Lefébure, médaille d'or à l'exposition de 1900 ", conservé à l'abbaye Notre-Dame d'Argentan, dans Mauboussin et Guillaumin, 2018, p. 54-55.

46. MAHB, DC00216.01.

47. MAHB, DC00273.01 et DC00273.02.

48. Dans ce dernier dessin, un picot est marqué sur chacun des côtés de quatre hexagones situés vers le centre du dessin ; on peut suivre le sens du travail en observant le sens des picots.

49. Le concours "Un des meilleurs ouvriers de France " (MOF) reprend cette terminologie du $\mathrm{XIX}^{\mathrm{e}}$ siècle, distinguant dans la classe «Dentelles » du groupe "Accessoires du vêtement » deux spécialités pour l'option 2 : "dentelles à l'aiguille : point d'Alençon » et "dentelles à l'aiguille : point d'Argentan " : voir par exemple sur le site internet du concours MOF (http://www-25.meilleursouvriersdefrance.org, consulté le 15 mars 2019) le « sujet des épreuves qualificatives " de la classe 01 du groupe 11, dans les « Archives du $24^{\mathrm{e}}$ concours (2013-2015). 
les Points de Venise. En effet, dans le Point de Venise, les motifs, larges et bordés d'un gros relief, ne se détachent pas sur un champ, mais sont reliés entre eux par de courtes brides (des barrettes) qui ont la forme d'un simple trait voire d'un "Y». Lorsque le champ se développe - en France - et que les motifs occupent relativement moins d'espace, le terme de « bride » est conservé ; mais cela peut sembler injustifié car les brides (les barrettes) se développent en triangles et hexagones irréguliers, puis en viennent à former des hexagones parfaitement réguliers. Lefébure rationalise le vocabulaire de la dentelle à l'aiguille au mépris de son histoire.

\section{Un point secret ?}

Le " Point " caractérisé techniquement comme " Point d'Argentan " par la maison Lefébure, est toujours pratiqué par les bénédictines de l'abbaye Notre-Dame. Dans un film réalisé en 2006, Une histoire de dentelle - Ville d'Argentan ${ }^{50}$, une religieuse, mère Colette, et trois anciennes élèves de l'école dentellière d'Argentan témoignent. Mère Colette explique que la technique du " Point " a été parfaitement conservée (depuis 1874) et transmise : "On avait vraiment la technique et on gardait la technique. Et je pense que c'est comme ça qu'on a réussi à le faire arriver jusqu'à maintenant " (10'54). " On a continué à faire toujours la dentelle, selon la tradition depuis Colbert, chez nous, entre nous » (12’02).

Bien plus, les religieuses détiendraient l'exclusivité de la technique du « Point d'Argentan » transmise par Désirée Hamel. Déjà dans les années 1930, cette technique était l'apanage des seules religieuses : elle n'était pas enseignée aux élèves. Yvonne Goulard explique ainsi : « on nous faisait faire des parties de dentelle mais pas de motif entier " (10’35); Suzanne Breistroff ${ }^{51}$ précise : " le coup de lancer la maille d'Argentan, on ne le connaissait pas ; c'étaient les religieuses qui le faisaient " (10’43) ; et selon Marcelle Toutain $^{52}$ : «si on peut le dire, c'est le secret du Point, ça » (10'49). Ce qui ferait la caractéristique du "Point d'Argentan " n'est même pas transmis aux novices ${ }^{53}$. Mère Colette précise : "On n'aime pas beaucoup parler de

50. Yolanda Communication, 2006 : https://www.dailymotion.com/video/xc56iy.

51. Élève à l'école dentellière de 1934 à 1939, entrée à l'âge de 14 ans.

52. Entrée à l'école dentellière à l'âge de 16 ans.

53. Comme nous l'a expliqué Mère Colette lors d'une visite organisée à l'abbaye par la Maison des dentelles d'Argentan, le 5 juillet 2018. 
secret d'ailleurs ; disons que c'est la technique " (11'04). Dans une autre interview elle explique ${ }^{54}:$ «seules les sœurs bénédictines de l'abbaye NotreDame d'Argentan détiennent le savoir-faire du Point d'Argentan. Il n'y a plus d'école ${ }^{55}$, ce qui n'empêche pas la transmission de la technique : [...] Tant que nous sommes en communauté, nous pouvons assurer le passage d'une génération à l'autre. " À une question concernant une éventuelle " appellation contrôlée ", elle répond à la journaliste : "Hélas, il n’y en a pas pour le Point d'Argentan, que seules garantissent les moniales et la mairie, qui les soutient ».

Pourtant, la mairie d'Argentan, après l'inauguration de la Maison des dentelles et du Point d'Argentan, le 21 juin $1997^{56}$, fait former quatre « dentellières en Point d'Argentan " - Véronique Thomazo est diplômée de cette première promotion ; puis un centre d'enseignement est attaché à la Maison des dentelles. Ces premières dentellières ont été formées par Marjolaine Salvador-Morel, ancienne élève de l'École nationale supérieure des Arts appliqués et des Métiers d'Art (ENSAAMA), par ailleurs titulaire du CAP «Arts de la dentelle, option aiguille ${ }^{57}$ ». Marjolaine SalvadorMorel a en effet pu consulter à Bayeux les archives de la maison Lefébure et elle y a découvert des échantillons pédagogiques. La pièce D1791, sur parchemin vert, conservée au Musée d'Art et d'Histoire Baron Gérard, montre sur cinq rangs la manière de réaliser ce que Lefébure appelle le "grand réseau d'Argentan".

La méthode enseignée par Marjolaine Salvador, comme celle des bénédictines d'Argentan, dépend donc de la réalisation par Désirée Hamel, ouvrière de la maison Lefébure, d'un « Point d'Argentan » ayant pour caractéristique un "grand réseau " que l'on appelait au XviII ${ }^{\mathrm{e}}$ siècle le " champ de bride ", à partir des échantillons retrouvés entre 1864 et 1874 « dans les greniers de l'hospice Saint-Louis ", l'ancien hôpital général d'Argentan ${ }^{58}$. L'une de ces pièces se trouve dans une salle de la Maison des dentelles

\section{Limon, 2015.}

55. L'abbaye est détruite par des bombardements en 1944. Lorsque les moniales hébergées à Sées rentrent à Argentan en 1958 dans l'abbaye reconstruite, il n'est plus possible d'ouvrir une école dentellière : selon mère Colette, "les charges étaient beaucoup trop fortes (11'54)".

56. Fouriscot et Styczynski, 1997, p. 30-32.

57. Audinet, 2000, extrait publié en ligne : http://www.patrimoine-normand.com/indexfiche-44379.html, consulté le 12 mars 2019.

58. L’hôpital général était sous l'Ancien régime le lieu d'enfermement des pauvres. 
d'Argentan ; une autre est accessible au public dans le cadre de l'exposition Jolies ornaises. Dentelles jumelles d'Alençon et d'Argentan ${ }^{59}$ (Fig. XXII et XXIII, cahier couleur).

Mais la technique reconstituée au XIX ${ }^{e}$ siècle par Désirée Hamel, est-elle la même que celle qui était employée au XVIII ${ }^{\mathrm{e}}$ siècle ? Pour répondre à cette question, nous disposons de deux types de documents datant d'avant la Révolution française : d'une part, des fameuses pièces provenant de l'hospice Saint-Louis que la dentellière a examinées ; d'autre part, d'une description détaillée de la technique du " champ de bride " que nous avons trouvée dans l'Encyclopédie méthodique (1785). Il s'avère que non seulement les deux types de documents concordent, mais qu'ils s'éclairent mutuellement.

\section{La technique du champ de bride au $\mathrm{XVIII}^{\mathrm{e}}$ siècle}

La description de la technique du "Point " (fait à l'aiguille) ne se trouve ni dans l'Encyclopédie $e^{60}$ de Diderot et d'Alembert ni dans la Description des arts et métiers ${ }^{61}$. Elle apparaît dans la section II "Des points et filets " de l'article «Dentelles, blondes \& points " de l'Encyclopédie méthodique de Roland de la Platière éditée par Panckoucke, et dans les planches qui l'accompagnent ${ }^{62}$. Ce volume ayant été publié en 1785, la technique décrite est bien celle qui avait cours avant la Révolution française et donc environ un siècle avant les recherches effectuées par Désirée Hamel pour la maison Lefébure en vue de retrouver la technique des « anciens points".

59. Voir ci-dessus.

60. Il n'existe pas d'article "Dentelle " dans l'Encyclopédie ou Dictionnaire raisonné des sciences, des arts et des métiers (ni dans le Supplément) ; le tome 12 de la première édition (Paris, 1765) contient toutefois un bref article " Point, (Dentelle) » qui mentionne pour les dentelles et passements faits à l'aiguille " les points de France, le point de Paris, le point de Venise, etc. », p. 875.

61. Dans le tome XIv des Descriptions des arts et métiers (1780), "Art de la lingerie par Mr de Garsault », ch. II, article 55, p. 125, la notice «Dentelles et points » ne comporte que quatre lignes. Le Point à l'aiguille est mentionné, mais non décrit, dans les articles " Dentelle » du Dictionnaire universel de Commerce de Jacques Savary des Bruslons (1723, 1741), du Dictionnaire portatif des arts et métiers (1766) de Philippe Macquer et de sa réédition augmentée (Dictionnaire raisonné universel des arts et métiers, 1773) par l'abbé Jaubert.

62. Publiées dans le Recueil de planches de l'Encyclopédie par ordre de matières, t. 6, 1786, article "Dentelle ", planche Iv, fig. 1 et fig. 2 [champ de réseau]; planche v, fig. 1 [champ de bride]. 
Le paragraphe I de la section II "Des points et Filets » de l'article «Dentelles, blondes et points » a précisément pour titre : «Point de France ou d'Alençon (Taenia linea opere Gallica ${ }^{63}$; manière de le faire) ${ }^{64}$ ". On notera qu'il n'est pas fait mention ici d'un "Point d'Argentan » : le titre de l'article montre que l'informateur qui a fourni les renseignements faisait un rapport sur le "Point de France " qui était produit dans la généralité d'Alençon, circonscription administrative dont dépendait l'élection d'Argentan.

L'auteur de l'article commence par exposer les phases préparatoires à l'exécution du "Point " : le report du dessin sur le vélin et le piquage : "Les dessins composés et choisis, se font graver communément sur cuivre, d'où on les empreint de noir sur du parchemin en veau bien préparé, auquel on a donné deux ou trois couches de teinture jaune faite avec de la graine d'Avignon ${ }^{65}$ ". Au XviII ${ }^{\mathrm{e}}$ siècle le parchemin est donc teint en jaune (et non en vert foncé comme il le sera à partir du XIX ${ }^{\mathrm{e}}$ siècle) ; le dessin est imprimé sur le vélin (tandis qu'au XIx ${ }^{\mathrm{e}}$ siècle il est piqué sur le vélin à travers un calque $^{66}$. En particulier, le dessin des hexagones représentant les mailles du champ de bride doit être bien visible sur la pièce, comme nous allons le montrer.

L'auteur précise la taille des morceaux de parchemin qui servent de support provisoire ${ }^{67}$. Le piquage, nécessaire pour maintenir le fil de trace à la surface du vélin, est effectué à l'aide d'un " poinçon ${ }^{68}$ ": on forme ainsi

63. Taenia linea opere Gallica se traduit littéralement «bande [en fil] de lin, travail français ».

64. Encyclopédie méthodique. Manufactures. Arts et métiers t. 1, 1785, p. 246-248.

65. La graine d'Avignon est la baie du rhamnus infectoria, le «nerprun des teinturiers " : voir l'Encyclopédie méthodique. Commerce, t. 2, 1783, p. 503, article "Graine d'Avignon » : "C'est la graine d'un arbrisseau connu des anciens sous le nom de Licium, de la Lycie où il croît en abondance, et aussi sous celui de Pizacanta, qui signifie bois épineux. Il y a quantité de ces arbrisseaux aux environs d'Avignon, d'où cette graine a été appelée graine d'Avignon [...] sa graine d'un vert tirant sur le jaune est de la grosseur d'un grain de froment, d'un goût astringent et amer [...] Quelques auteurs confondent cet arbrisseau avec le noir prun [nerprun], mais il est d'une espèce toute différente. Les teinturiers se servent de la graine d'Avignon pour teindre en jaune. »

66. Voir Odolant-Desnos, 1942, p. 1.

67. Ils sont " hauts de trois à quatre doigts " et leur longueur "varie depuis cinq jusqu’à douze pouces ". Une paire de manchettes d'hommes, par exemple, se compose de dix morceaux qui sont assemblés.

68. Ou bien d'une « longue aiguille moyenne ». Selon l'auteur, on peut piquer plusieurs morceaux à la fois en les superposant. 
"de petits trous espacés d'une ligne ${ }^{69}$ sur tous les contours des fleurs". On procède ensuite au montage du vélin sur une toile pliée en deux ${ }^{70}:$ les trois couches sont cousues ensemble à grands points ${ }^{71}$. L'auteur explique comment on fixe le fil de " trace " qui servira de cadre et de support pour l'exécution des motifs ${ }^{72}$. Il précise aussi la manière de tenir l'ouvrage et l'aiguille : "L'ouvrage se tient de la main gauche de manière que l'index soit dessous, et le pouce en-dessus avec le doigt medius : l'aiguille est tenue entre l'index et le medius de la main droite; le pouce revêtu d'un doigtier de peau, demeure libre pour la diriger ». Le travail se fait donc avec l'aiguille pointe en l'air.

La manière d'exécuter le " toilé qui remplit les fleurs " est détaillée ${ }^{73}$ et l'auteur apporte ici une précision qualitative intéressante : " ce point s'exécute dans toute sa perfection aux environs d'Argentan et d'Alençon ; c'est dans ces campagnes qu'on le fait faire ; il est d'une finesse et d'une beauté auxquelles l'extrême blancheur prête encore son éclat ${ }^{74}$ ".

C'est alors qu'il est question des fameux « champs », que Lefébure appelle " petit réseau » et " grands réseaux ", et qui permettraient selon lui de caractériser et de distinguer le Point d'Alençon, le Point d'Argentan et le Point

69. La " ligne" est ici une unité de longueur : voir Descriptions des arts et métiers, t. XIv, 1780, "Art de la lingerie par Mr de Garsault », ch. I, «L'aune et ses fractions réduites en pieds, pouces et lignes ", p. 118-120.

70. "On applique chaque portion de parchemin sur un semblable morceau de grosse toile écrue, que l'on met en double".

71. Voir la représentation de ce fil de fixation dans le Recueil de planches, t. 6, «Dentelle » pl. 4.

72. «On prend deux fils plats à rentraire (il s’agit d'un fil employé pour réparer les déchirures dans une étoffe (voir la Descriptions des arts et métiers, t. XIV, 1780, "Art du tailleur par Mr de Garsault", ch. VI, "Points à rabattre et de rentraiture », p. 66) que l'on maintient sous le pouce gauche, en les conduisant sur toute la suite du dessin, et on les fixe avec du fil rond enfilé dans une aiguille qu'on fait passer d'abord de dessous en-dessus, dans un des trous du piqué, et qu'on retire de dessus en-dessous, après l'avoir fiché dans le même trou, en faisant embrasser les deux fils plats sous le point qu'on forme ainsi, et qui sert à les arrêter " (Encyclopédie méthodique. Manufactures, t. 1, p. 246-247). Autrement dit, en suivant le contour des motifs, on tend un double fil de trace à la surface du vélin, et on le maintient en place à l'aide d'une aiguillée de fil qui entre et sort par chacun des trous préalablement percés avec le poinçon, tout en traversant les trois épaisseurs de vélin et de toile. Le fil de trace ne traverse en aucun cas le vélin ; il est maintenu à la surface.

73. On fait des rangs de points noués (il s'agit de points de feston qui prennent appui sur la trace puis sur les rangs déjà exécutés) en travaillant toujours de gauche à droite ; au bout de chaque rang on arrête le fil à la trace, on le rejette à gauche, et on commence un nouveau rang en faisant des points noués sur ce fil tendu, tout en faisant rentrer l'aiguille dans chacun des points du rang précédent.

74. Encyclopédie méthodique. Manufactures, t. 1, p. 247. 
de France. Or d'après l'Encyclopédie méthodique « le champ est de bride ou de réseau ». L'auteur explique que le réseau " ne présente que des mailles simples comme celle du filet le plus fin et le plus serré ${ }^{75}$ ». Les mailles du réseau sont petites, de forme rectangulaire et elles ne sont pas recouvertes d'un autre point ${ }^{76}$.

L'auteur en vient alors à la réalisation du fameux " champ de bride ${ }^{77}$ ». Il explique que la bride " est une figure à six pans " - autrement dit, de forme hexagonale. Il ajoute qu'elle " est toujours marquée sur les dessins ${ }^{78}$ ». Le travail de l'ouvrière est ainsi guidé par le dessin des hexagones imprimé en noir sur le parchemin teint en jaune.

Un autre point remarquable est ici l'utilisation d'épingles pour réaliser la bride : " avant de la commencer on la pique dans toute l'étendue du champ, mais seulement à l'angle supérieur de chaque hexagone ${ }^{79}$ ». Ensuite " on attache son fil au bord d'une fleur à gauche, on passe l'aiguille dans la lisière ; puis, mettant une épingle dans le trou formé à l'angle supérieur de

75. Pour exécuter le réseau " on commence par jeter un fil de champ : ce fil est comme la chaîne de l'ouvrage " [en effet, à l'inverse de la bride, le réseau est fait de rangs perpendiculaires par rapport au pied de la dentelle], " on l'attache de part et d'autre à la trace, et on le recouvre d'un autre qui achève de former les mailles".

76. Voir leur forme dans la planche iv de l'article «Dentelle», dans le tome 6 du Recueil de planches, qui montre selon l'" Explication des planches ", " un patron de Point proprement dit ». Il est précisé dans cette "Explication des planches " (Encyclopédie méthodique. Manufactures, t. 1, p. 251) que les "barres transversales du milieu sont de simples rayures du dessin destiné à guider, dans l'exécution du champ".

77. Ce texte est copié par madame Celnart, au début du XIX siècle, dans son Manuel des demoiselles (Celnart 1826, p. 159-163 et Celnart, 1828, p. 128-130. Élisabeth Celnart explique que ne sachant pas faire de Point d'Alençon elle a dû avoir recours à des notions étrangères : " on m'a fourni la note suivante " qu'elle intitule : «Point d'Alençon d'après Rolland ». Le texte est un extrait abrégé de ce qui a été publié par Roland de la Platière (et non « Rolland " dans la première édition du Manuel, voire «Rollant " dans la troisième), dans le paragraphe consacré au " Point de France ou d'Alençon " et à la "manière de le faire ". Le dessin de la figure 63 de la planche II du Manuel des demoiselles est une copie assez fidèle d'un détail de la figure 2 de la planche IV (consacrée à la "Dentelle »), du t. 6 du Recueil de planches.

78. La figure 1 de la planche $\mathrm{v}$, qui l'illustre, porte l'explication suivante : " patron en parchemin de Point, dont le champ doit être de bride " : voir "Explication des planches ", Encyclopédie méthodique. Manufactures, t. 1, p. 251 et Recueil de planches, t. 6, art. "Dentelle", planche v.

79. Encyclopédie méthodique. Manufactures, t. 1, p. 247. 
la bride, on passe le fil autour, et l'on suit ainsi jusqu'à la première fleur ${ }^{80}$ à droite où l'on arrête son fil qui forme alors une rangée en zigzag ${ }^{81}$ ".

Mais que signifie donc " on passe le fil autour " de l'épingle fixée sous le sommet de l'hexagone ? Pour bien comprendre cette étape, il nous faut retourner au MAHB de Bayeux : Désirée Hamel en observant, voire en démontant un peu, les pièces anciennes non achevées trouvées dans le grenier de l'hospice Saint-Louis avait compris que l'armature interne de la bride était une maille à double boucle, la même que celle du réseau, mais d'une taille plus grande. Et c'est bien en raison de la similitude de ces mailles que Lefébure parle de " petit » et de " grands réseaux " au lieu de " champ de réseau " et de " champ de bride ". Le point exécuté sur parchemin vert, dans la pièce D1791 du MAHB (Fig. XXIV, cahier couleur), montre sur cinq rangs partiellement recouverts de points noués (festons simples) que l'armature sous-jacente se compose de points de feston espacés, où le fil est enroulé deux fois : c'est cette double torsion qui confère la hauteur souhaitée aux deux pans verticaux de l'hexagone ${ }^{82}$.

Le rang de retour (de droite à gauche) est bien expliqué dans l'Encyclopédie méthodique ${ }^{83}:$ «on revient sur cette rangée en repassant les épingles, et l'on réunit avec l'aiguille les parties de fil qui forment le zigzag ${ }^{84}$ ». L'épingle maintient toujours la maille en place par son sommet. À partir du deuxième rang " on passe le fil à chaque rangée dans la pointe de celle qui précède "; les hexagones sont dès lors bien formés.

80. Il est en effet difficile d'assembler deux pièces par des brides; il est plus aisé de terminer le rang sur un motif comportant un fond.

81. De même, dans l'" Explication des planches » l'auteur précise à propos de la figure 1 de la planche v (art. «Dentelle»), qui représente un « patron en parchemin de Point, dont le champ doit être de bride » : «La bride est toujours dessinée sur le patron ; pour la faire on commence par piquer tous les hexagones qu'elle forme, à leur angle supérieur ; comme on voit par les petits trous noirs qui représentent les trous faits par le piquage » [en réalité, le dessinateur de la planche les a omis]. "On met des épingles dans ces jours, à mesure qu'on travaille, pour tourner les fils autour, et déterminer les contours qu'on veut lui donner" (Encyclopédie méthodique. Manufactures, t. 1, p. 251 ; voir la planche v, dans le Recueil de planches, t. 6, art. "Dentelle ", sans pagination).

82. On peut voir l'exécution de ce type de point dans une vidéo de l'Atelier National du Point d'Alençon : Le savoir-faire de la dentelle au point d'Alençon, réalisé par Dino Gambini et produit par André Guéret, Atelier Cinéma Normandie, ACCAAN, 2003 (version courte, mn. 5’26 à 5’40) : https://www.youtube.com/watch?v=M8tNgeG3FPA (consulté le 15 mars 2019).

83. Alors qu’il est absent dans la pièce conservée à Bayeux.

84. En effet, c'est ce rang de retour qui permettra de bien former les hexagones : on n'enroule ici le fil qu’autour des deux côtés supérieurs de la maille hexagonale (ce qui forme la ligne en «zig-zag ») en serrant bien lorsque l'on passe d'une maille à l'autre. 
Une fois cette armature entièrement mise en place sur l'ensemble d'un espace délimité par des motifs, la deuxième étape du travail peut commencer : "Quand la figure de la bride est ainsi terminée, on recouvre le tout d'un point noué fait avec du fil très fin, au nombre de sept à huit points très serrés sur chaque pan de la bride ». Autrement dit chaque côté de la maille hexagonale est recouvert de sept à huit points de feston ${ }^{85}$.

La pièce provenant du grenier de l'hospice Saint-Louis, que nous avons pu observer de près lors de l'exposition Jolies ornaises, présente exactement les mêmes caractéristiques (Fig. XXIII, cahier couleur) : parchemin de couleur claire sur lequel le dessin est entièrement imprimé en noir, y compris le dessin des mailles hexagonales; traces des trous d'épingle juste sous l'angle supérieur de chaque bride (ou maille hexagonale). Elle permet aussi de comprendre quel est le sens du travail lors de la deuxième étape : les mailles sont festonnées rang par rang, de gauche à droite ${ }^{86}$.

Après avoir traité des champs, l'auteur aborde les « modes » (ou " points de fantaisie »), "le brode " (le léger relief qui borde les fleurs), puis la manière de détacher du parchemin la pièce terminée, le passage de la dent de loup pour lustrer l'intérieur des motifs, et l'assemblage des différents morceaux.

\section{Conditions sociales de la fabrication du « Point » au XVIII ${ }^{\mathrm{e}}$ et au XIX ${ }^{\mathrm{e}}$ siècles}

Après ces explications techniques, l'auteur de l'article « Dentelles, blondes et points " de l'Encyclopédie méthodique publie un rapport sur les conditions sociales du travail du "Point». Ce travail est divisé entre plusieurs personnes :

on y emploie des enfants dès l'âge de six ans : soit à la trace, soit au champ de bride, soit au fond, les ouvrières de ces trois classes continuent assez généralement le reste de leur vie le genre unique de travail dans lequel elles ont été instruites. Il en est peu dont l'intelligence et l'adresse soient telles qu'elles les fassent parvenir

85. Une méthode plus rapide et moins coûteuse en main d'œuvre consiste à tortiller (surjeter) le fil autour de la bride, au lieu de la festonner.

86. Pour travailler le pan vertical de la bride, le fil est amené du bas vers le haut du côté, et fixé à l'intersection des fils; ce côté est alors festonné en descendant. Le festonnage prend donc la forme d'un rang de " $U$ » joints les uns aux autres (il n'y a pas de rang retour). 
à fabriquer le « Point » en totalité ; ce sont celles de goût et de confiance qui règlent et perfectionnent le travail, en réunissant les morceaux de "point ", selon la suite du dessin ${ }^{87}$.

Le " Point " nécessite donc de nombreuses opérations (trace, bride ou réseau, fond, rempli, modes, brode), qui sont toutes effectuées à la main, et le travail est divisé entre plusieurs ouvrières très spécialisées qui opèrent tour à tour sur la même pièce. Seules les ouvrières qui assemblent les morceaux connaissent tous les points. Les fabricants marchands, avec privilège royal ou non, possèdent des bureaux dans diverses localités de la généralité d'Alençon ; ils fournissent la matière d'œuvre aux ouvrières, qui travaillent dans le cadre d'une manufacture "dispersée ${ }^{88}$ " (bien qu'il existe quelques écoles ouvertes par des religieuses et des ateliers dans les hôpitaux généraux). Le travail s'effectue principalement à domicile ${ }^{89}$, aussi bien en ville qu’à la campagne. En milieu rural, l'activité dentellière à temps partiel complète le temps de travail agricole, notamment l'hiver.

On notera que l'exécution du " champ de bride " est demandée à des enfants dès l'âge de six ans, et aux ouvrières les moins douées. Il ne s'agit pas d'une technique réservée à des expertes. À l'hôpital général, lieu d'enfermement des pauvres et des enfants des "hérétiques " (protestants) de basse extraction ${ }^{90}$, on y emploie des filles et des garçons ${ }^{91}$. Une liste de fillettes, et d'adultes, ouvrières de Jeanne James, directrice d'une manufacture de Point de France à Argentan, et datée du 23 novembre 1744, a été publiée par Gérard Kempf ${ }^{92}$.

87. Encyclopédie méthodique. Manufactures, t. 1, p. 247-248.

88. Lenhof, 2009, notamment p. 239-241.

89. Les ouvrières avaient rapidement obtenu le droit de travailler chez elles, et non au bureau de la manufacture, contrairement à que préconisait l'arrêt du Conseil d'État du 5 novembre 1665 dans le cadre du privilège de dix ans, accordé à la manufacture des Points de France : voir Despierres, 1886, p. 25-27. Les jeunes filles pouvaient être mises en apprentissage au domicile des maîtresses dentellières : voir le fac-similé de contrats d'apprentissage dans Million, 2001, p. 53 et 55. Voir aussi Poupet, 1913, p. 75 et p. 86-88.

90. Capul, 1989 et 1990. Sur Louis-François Lallemant de Lévignen, intendant d'Alençon, qui gérait les demandes de privilège des manufactures de « Point », voir Kempf, 1997, p. 5 et p. 16-17 et Kempf, 2010, p. 4-8 et p. 9-10. Le même intendant faisait enlever les enfants des protestants et envoyait ceux de basse extraction à l'hôpital général - où l'on fabriquait de la dentelle : voir Clolus, 2006.

91. Poupet, 1913, p. 78-82.

92. Kempf, 1997, p. 13-14. 
Il n'y a pas de dentellières "à leur compte ${ }^{93}$ ». Les ouvrières sont rémunérées à la tâche ; leur salaire est précisé dans le document : elles "gagnent par jour depuis trois sols jusqu'à trois livres. Les prix varient en raison de l'espèce de travail, de la qualité des ouvrages et des talents de chacune".

L'auteur note toutefois que les prix ont baissé "d'environ un tiers depuis dix-huit mois "; "malgré la perfection du "Point", son commerce et son prix diminuent considérablement de jour en jour ${ }^{94}$. Le rapport nous renseigne aussi sur le prix des fils, particulièrement onéreux, achetés à la fabrique de Lille, et sur la valeur d'une manufacture : «La manufacture du "Point" de France peut être évaluée à 1200000 livres, dans lesquelles il entre au plus pour 150000 livres de fils de Flandres : elle occupe au moins huit à neuf mille ouvrières, tant d'Alençon, que de trois à quatre lieues à la ronde ». "Il se fabrique la même espèce de "point" dans la ville d'Argentan, d'où ce travail prend le nom de "point" d'Argentan : il entre dans les assortiments de commerce du "point" d'Alençon, auquel il est supérieur en finesse et en perfection. L'objet de cette fabrique est d'environ 500000 livres ». Gérard Kempf cite pour sa part la dernière portion de ce document à partir des rapports manuscrits rédigés en 1772 et en 1777 par le sieur Brunet ${ }^{95}$, inspecteur des manufactures de la généralité d'Alençon ${ }^{96}$.

Ce document confirme qu'au XviII ${ }^{\mathrm{e}}$ siècle ce ne sont pas des styles de champ qui permettent de distinguer le Point d'Argentan et le Point d'Alençon, mais plutôt leur provenance géographique au sein de la généralité d'Alençon, et la supériorité qualitative des produits d'Argentan sur

93. Il n'existe pas d'ouvriers-marchands de dentelle, contrairement à ce qui se passe en région parisienne pour la dentelle aux fuseaux : $c f$. Buffévent, 1984, p. 117.

94. Encyclopédie méthodique. Manufactures, t. 1, p. 248.

95. Kempf, 1997, p. 3 ; selon Gérard Kempf ces rapports datent du 3 février 1772 et du 5 décembre 1777. La cote du document (Archives nationales KK1713) est toutefois erronée. Le rapport de l'inspecteur Brunet ne se trouve pas non plus sous la cote KK1317 (mentionné dans Plessix (dir.), 1999, p. 191) ; le "Mémoire de la généralité d’Alençon dressé par Mr d'Argenvilliers intendant de la généralité " présent sous cette cote KK1317, contient bien un paragraphe sur les " manufactures des Points de France ", mais il date de la fin du XviI siècle. Nous poursuivons nos recherches pour retrouver le rapport du sieur Brunet. Roland de la Platière était lui-même inspecteur des manufactures.

96. Brunet est inspecteur des manufactures de la généralité d'Alençon de 1757 à 1783 : voir Baquié, 1927, p. 367. Sur les mémoires des inspecteurs des manufactures, voir aussi Minard, 1994 ; sur la publication des rapports dans la Méthodique, voir p. 523-526 ; certains inspecteurs sont également auteurs de traités techniques. 
ceux d'Alençon, comme l'a par ailleurs montré Gérard Kempf, à partir de documents conservés dans les Archives départementales de l'Orne ${ }^{97}$.

Lauteur, qui rend public le rapport de l'inspecteur Brunet dans l'Encyclopédie méthodique, nous apprend encore que le champ de bride (plus solide ${ }^{98}$ ) est préféré en France, et le champ de réseau (plus séduisant) à l'étranger. Enfin, il rappelle le lien fort entre le " Point » et la maison du roi de France : " c'est du point d'Alençon et d'Argentan que l'on décore les lits et les toilettes des Dames de France et des Princesses du Sang dans les cérémonies de mariage, des couches et des baptêmes ; ces sortes de parures valent depuis mille écus jusqu’à 30 et 40000 livres. Les jours de cérémonie le Roi porte encore des cravates; elles sont du plus beau point ». La "Manufacture des Poincts de France " avait en effet été fondée à l'instigation de Colbert le 14 août 1665 par une " Déclaration du Roy » enregistrée en Parlement ${ }^{99}$ : il s'agissait, au XVII ${ }^{\mathrm{e}}$ siècle, d'empêcher la noblesse d'acheter des dentelles à l'étranger, notamment à Venise. Pendant des décennies, la famille royale et la cour avaient dû porter du Point de France, mais la reine Marie-Antoinette ne l'appréciait plus et s'était libérée de cette contrainte. Un dossier conservé aux Archives nationales contient la supplique d'un avocat, échevin de la ville d'Alençon, Olivier de Saint-Vast, envoyée le 2 août 1780 à monsieur de Tolozan et adressée au Directeur général [des finances] ${ }^{100}$ : en s'appuyant sur le rapport du sieur Brunet, le notable déplore la chute des manufactures de Point de France :

Comme rien ne vous échappe et que le commerce et les manufactures ont mérité votre attention, $\mathrm{Mr}$ Brunet notre inspecteur que vous avez mandé auprès de vous, vous a sans doute instruit que la fabrique de point d'Alençon, qui faisait vivre les deux tiers de la ville, est totalement tombée, depuis que notre auguste reine, n'a pas jugé à propos d'en porter. Par le discrédit de la fabrique, et ne s'en faisant point de consommation à l'étranger à cause de la guerre, dix mille personnes dans la ville d'Alençon sont dans la misère, ce que j'ai aperçu par le recensement que j'ai fait depuis deux ans avec le maire de la ville. La même chose est arrivée à Argentan et autres endroits circonvoisins. Ne serait-il

97. Kempf, 1997, p. 1-32 et Kempf, 2010.

98. Encyclopédie méthodique. Manufactures, t. 1, p. 247.

99. Voir la reproduction de ce texte dans Million, 2001, p. 37-39.

100. Tolozan est intendant du commerce entre 1776 et 1791 : voir Garrigues, 1998, p. 626-661. 
point possible, Monseigneur, que vous missiez sous les yeux de sa majesté ces justes représentations, et que la reine par commisération daignât porter du point d'Alençon seulement une fois par semaine en coiffure, mantelet, ou garniture de robes, ce serait faire revivre le commerce et rendre la vie au moins à plus de cinquante mille personnes, qui sont dans la misère, ne gagnant par jour que deux et trois sols, au lieu de vingt et vingt-cinq sols que leurs journées leur produisaient auparavant ${ }^{101}$.

La réponse brève, datée du 17 septembre 1780, est dénuée de compassion pour la misère des dentellières et la ruine des marchands : le "Point » est, comme bien d'autres objets, "soumis à l'empire des modes " et " conséquemment sujet à des révolutions »; ces changements sont sans doute " un malheur pour les fabricants et Marchands » dont le travail diminue ; mais on ne peut « sur cela gêner le goût du consommateur, et encore moins celui de la Reine ».

Ce n'est donc pas la Révolution française qui a fait péricliter les fabriques de Point, mais plutôt la mode changeante promue par la reine à la cour. En 1780 les manufactures de Point de France fondées sous un régime protectionniste au XVII ${ }^{\mathrm{e}}$ siècle étaient complètement " tombées ".

\section{De l'industrie de luxe, sensible aux modes, à l'œuvre d'art pérenne}

Sous l'Ancien régime, le "Point ", à l'aiguille, était destiné à la famille royale et à la noblesse, dans le cadre d'un patriotisme économique. Après une éclipse de plusieurs décennies, la fabrication du " Point " aurait sérieusement repris à Alençon vers $1846^{102}$. Sous Napoléon III, cette industrie redevient florissante. Les consommateurs de dentelle à l'aiguille sont maintenant des bourgeois. Un acteur supplémentaire intervient au-dessus du marchand fabricant : les commandes lui sont passées par des maisons de négoce en articles de Paris : «Les gros profits d'ailleurs sont absorbés par les marchands de Paris, et le pays producteur n'y a pas une part suffisante ${ }^{103}$ ». Les marchandises sont exportées en Europe et jusqu’en Amérique.

101. Archives nationales F 12/561, dossier 2.

102. Lefrou, 1858, p. 286.

103. Ibid., p. 287. 
Dès 1855 , on comprend qu'à la différence de la dentelle aux fuseaux, qui peut être correctement imitée par la mécanique, il est impossible de réaliser le " Point » à la machine ${ }^{104}$. Le travail, toujours divisé, continue à être effectué dans le cadre de la manufacture dispersée "faisant fabrique mais n'ayant pas de réunion d'ouvriers ${ }^{105}$ ": un rapport de J. Odolant-Desnos daté de 1842 précise qu’à cette date, seize opérations sont exécutées « séparément par une ouvrière spéciale ». Mais la technique du champ de bride dans la fabrique de la maison Docagne (ou d'Ocagne) à Alençon n'est pas, en 1842, celle que décrivait l'Encyclopédie Méthodique en 1785. Une modification importante est intervenue : pour effectuer la bride, la première opération consiste à coucher des fils qui vont lui servir d'armature, c'est-àdire à les tendre à travers tout le champ :

Pour établir la bride on « couche " des fils, d'abord de gauche à droite, puis de droite à gauche, sur la bande de parchemin [...] Pour donner plus de force à ces mailles de la bride, représentées alors seulement par les vides que les points d'intersection des fils de la " couchure " laissent entre eux, on entourait autrefois chacun des six côtés que doit avoir la maille de nœuds bouclés semblables aux points de feston, et nous avons vu chacun de ces côtés d'une maille, n'ayant que deux millimètres de diamètre, recouvert de dix et même de douze nœuds bouclés ; cela constituait la « bride festonnée ». L'on fit ensuite la "bride bouclée", dont les six coins seuls de chaque maille sont arrêtés par quelques nœuds bouclés ; d'autres fois, si l'on tient à une très grande régularité dans les mailles, on maintient les vides de la couchure par des épingles, ce qui produit la " bride aux épingles ». Mais le plus souvent, pour donner à la dentelle un prix moins élevé, on se contente de faire "une bride coulée ", et alors chacun des côtés des mailles est simplement entouré d'un fil très légèrement arrêté par un seul nœud bouclé dans les coins, et ce genre de mailles fait la bride sinon la plus solide, du moins la plus légère ${ }^{106}$.

Alençon conserve donc le terme ancien de «bride " mais modifie complètement la manière de faire l'armature. L'auteur explique ensuite que si l'on

104. "On a également essayé de la mécanique, sans plus de succès, et l’on s'accorde à donner la préférence au point fait à la main ": Cussy, 1855, p. 267.

105. Lenhof, 2009, p. 239-241.

106. Odolant-Desnos, 1842, p. 1. 
veut donner encore plus de légèreté à l'ouvrage, on remplace la bride par du réseau.

Cette méthode est aussi attestée dans la manufacture Huignard à Alençon ${ }^{107}$, et c'est celle que décrit Madame Despierres dans son Histoire du Point d'Alençon ${ }^{108}$, en ignorant la technique ancienne décrite dans l'Encyclopédie Méthodique. Or cette technique par couchure semble inspirée du fond de tulle de la dentelle aux fuseaux. En effet J. Odolant-Desnos rapporte que dès 1834 des expériences techniques avaient été menées pour remplacer le champ "par un tulle en fil fait au métier " sur lequel on aurait brodé des motifs à la manière du Point d'Alençon. En 1840 un fabricant aurait pris un brevet pour remplacer l'armature du champ de bride par un tulle de coton réalisé par un procédé mécanique, ce tulle étant ensuite festonné ${ }^{109}$. Cette tentative de mécanisation serait restée sans suite.

Une autre modification tient à l'utilisation du fil de coton (dont la production mécanisée a beaucoup progressé) à la place du fil de lin, sauf éventuellement pour l'exécution du champ ${ }^{110}$ : avec l'emploi du fil de coton, le travail devient plus aisé pour les ouvrières. Quoique moins solide, la dentelle a beaucoup gagné en grâce et en légèreté ${ }^{111}$. On s'adapte aux goûts d'une clientèle bourgeoise et l'on produit aussi des pièces de petite dimension - comme des cols - qui sont moins onéreuses que les longs volants ${ }^{112}$. Enfin le dessin n'est plus imprimé sur la pièce à l'aide d'une presse : «la décadence progressive de l'industrie dentellière fit, petit à petit, abandonner les presses. On se contente actuellement de décalquer à l'encre sur

107. Voir la photographie d'un échantillon conservé à Alençon au Musée des Beaux-Arts et de la dentelle, dans le catalogue de l'exposition Jolies ornaises (Mauboussin et Guillaumin, 2008, p. 12 et p. 57. Voir aussi Musée des Beaux-Arts et de la dentelle (dir.), 2011, p. 34-35.

108. Despierres, 1886, p. 80-83. Elle est reprise par Boulard, 1924, p. 57-58. On la trouve aussi dans le catalogue de l'exposition Jolies ornaises, p. 12.

109. Odolant-Desnos, 1842, p. 1.

110. L'utilisation du coton à la place du fil de lin est l'objet d'un débat dont fait état l'Annuaire des cinq départements de l'ancienne Normandie, 1858, p. 302-303.

111. L'Annuaire des cinq départements de l'ancienne Normandie, 1858, p. 302-303. En 1855, le vicomte de Cussy notait : "Le point d'Alençon actuel n'est pas tout-à-fait ce qu'était l'ancien ; il est plus léger, mais n'en est pas moins encore fort riche »: Cussy, 1855, p. 267.

112. Lefrou, 1858, p. 285. 
des papiers transparents, les dessins établis d'abord au crayon ". Le dessin est dessiné sur du papier et piqué sur le parchemin à travers un calque ${ }^{113}$. À la fin du XIX ${ }^{\mathrm{e}}$ siècle il s'agit de maintenir à tout prix, dans une compétition effrénée ${ }^{114}$, le « Point » fait entièrement à la main contre l' « imitation » mécanique de la dentelle aux fuseaux, produite notamment dans le nord de la France. Fernand Engerand déplorera, dans la Revue des deux mondes, en 1900, la brutale invasion des machines dans le domaine de l'art, la "vulgarisation de la mode sous l'influence de nos mours égalitaires et démocratiques ${ }^{115}$ »; selon lui, la véritable dentelle va de pair avec le régime monarchique ${ }^{116}$. La dentelle à l'aiguille devient un article de très grand luxe qui permet aux membres de familles royales européennes de se distinguer, le Point fait à la main étant " regardé comme de suprême distinction ${ }^{117}$ ". Le prix " énorme " du " Point ", " assure aux riches élégantes le privilège de seules pouvoir en porter ${ }^{118}$ ». Un incomparable rochet en dentelle à l'aiguille est offert par le diocèse de Bayeux au pape Léon XIII pour son jubilée en $1887^{119}$. La maison Lefébure invente même un nouveau champ : celui-ci comporte des « petits rinceaux s'enrouant comme les filigranes des bijoux d'or qu'on fabrique en Orient ${ }^{120}$ ».

La dentelle à l'aiguille dont la technique et les dessins sont perfectionnés à l'extrême ${ }^{121}$, tient maintenant "bien plus de l'art que de l'industrie ${ }^{122}$ ". Comme elle est passée au rang d'œuvre d'art, elle doit s'exposer dans un espace muséal : Ernest Lefébure propose d'ouvrir à Bayeux un " musée spécial ». Des vitrines contenant des échantillons de dentelle de Bayeux de

\footnotetext{
113. Boulard, 1924, p. 88. Mais pour la bride par couchure, les hexagones sont dessinés sur le parchemin : voir Mauboussin et Guillaumin, 2008, p. 12 (photo).

114. Voir Lefébure, 1913, p. 48-49 et p. 52-54. La maison Lefébure participe à toutes les compétitions et expositions de prestige en Europe et en Amérique, en vue de remporter les prix d'excellence. 115. Engerand, 1900, p. 647.

116. Ibid., p. 648.

117. Cussy, 1855, p. 267.

118. Odolant-Desnos, 1842, p. 2.

119. Lefébure, 1813 , p. 56-57 et p. 49-51.

120. Ibid., p. 58.

121. Ibid., p. 39 : « jamais à aucune époque on n’avait rien vu d'aussi réellement artistique que ces points d'Alençon, provenant de Bayeux!».

122. Lefébure, 1813, p. 54 ; comparer avec Cussy, 1855, p. 265-266.
} 
toutes les époques sont installées « dans une des salles de la Bibliothèque, à côté de la Tapisserie de la Reine Mathilde ${ }^{123}$ ».

Par ailleurs, d'un point de vue social, l'industrie dentellière au XVIII ${ }^{\mathrm{e}}$ siècle avait pour vertu de faire "subsister un grand nombre de familles " et de " retirer du libertinage une quantité de filles que la misère ou l'oisiveté ne manquerait pas d'y plonger ${ }^{124}$. En 1858 , le "Point " occuperait à Alençon douze à treize cents femmes : dont six à sept cents dans la ville, plusieurs centaines dans des communes à l'ouest d'Alençon et dans le canton de Carrouges, et cinq à six cents paysannes " qui gagnent à faire $\mathrm{du}$ point plus que leurs pères, frères ou maris, ne gagnent aux travaux du pays ${ }^{125}$ ". Il s'agit à l'aube du $\mathrm{xx}^{\mathrm{e}}$ siècle de maintenir, contre l'usine, l'« industrie domestique » dans laquelle «l'ouvrière ne loue pas son temps, et n'aliène pas sa liberté ", " peut varier ses occupations ", prendre ou quitter son travail selon sa volonté, conservant « toujours la plus absolue liberté d'action ${ }^{126}$. La dentelle est considérée par F. Engerand comme une " occupation saine » qui " retient femmes et enfants à la maison, évite la dissipation ${ }^{127}$. L'activité dentellière permettrait aussi de lutter contre l'exode rural, le risque de désœuvrement et d'alcoolisme dans les campagnes : selon F. Engerand, c'est le moyen d'enrayer la " désolante dépopulation des campagnes en permettant à la paysanne de gagner sa vie chez elle et d'apporter un appoint sérieux au salaire du mari ; ainsi elle retient, elle fixe la femme au village, et où est la femme est le foyer; au point de vue social, son importance est extrême ${ }^{128} »$. Ce sont bien les vertus morales de

123. Lefébure, 1813, p. 57. La demande en avait été faite dès 1857 par le marquis de ChennevièresPointel : voir l'Annuaire des cinq départements de l'ancienne Normandie, 1856, p. 132 et par M. de Caumont en 1855 et en 1860, "Utilité des musées spéciaux et des expositions d'art et d'industrie ", Annuaire, 1856, p. 51 et p. 75-76.

124. Voir la lettre du sieur Boirel, subdélégué à Argentan, écrite en 1741 à l'intendant d'Argentan, M. Lallemant de Lévignen, et publiée par Kempf, 1997, p. 15. En 1727 un rapport, publié dans le Bulletin de la Société historique et archéologique de l'Orne, 1898, p. 52, décrivait les difficultés de la manufacture royale de Montulay (1714-1740) à Argentan : "Cette manufacture, dans le temps qu'elle était en vigueur, entretenait 7 à 800 ouvriers de la ville et des environs. On y faisait travailler des enfants à l'âge de sept à huit ans, ce qui les accoutumait de bonne heure au travail et leur faisait gagner de quoi subsister. Mais à présent une partie des filles qui étaient employées à ces travaux, manquant d'ouvrage et réduites à l'oisiveté, se donnent au libertinage et à la débauche ".

125. Annuaire des cinq départements de l'ancienne Normandie, 1858, p. 285-286. Le salaire des dentellières est indiqué dans ce document.

126. Lefébure, 1913, p. 44.

127. Engerand, 1900, p. 646.

128. Ibid., p. 662. 
ces " produits tous fabriqués dans la famille " qui, à la veille de la première guerre mondiale, motivent ecclésiastiques et notables à soutenir l'activité dentellière ornaise, à en écrire l'histoire et à promouvoir celle-ci à travers le Bulletin de la Société historique et archéologique de l'Orne ${ }^{129}$.

En conclusion, l'Encyclopédie méthodique est une source très précieuse et méconnue pour la reconstitution de la technique du "champ de bride " tel qu'il était exécuté au XVIII ${ }^{\mathrm{e}}$ siècle, avant la disparition du "Point royal de France ", auquel la reine Marie-Antoinette préférait la gaze et le tulle. Il s'avère que l'exécution en était assez aisée, puisqu'elle était confiée à des ouvrières qui n'étaient pas les plus douées en "intelligence " et en " adresse », et même à des enfants, dès l'âge de six ans. Il ne s'agissait en aucun cas d'une technique réservée à des expertes. Mais il est vrai qu'en imprimant tout le dessin en noir sur un parchemin teint en jaune, et en utilisant des épingles pour donner une forme hexagonale à la maille et favoriser sa régularité, la difficulté d'exécution n'était pas très grande ${ }^{130}$. Un vocabulaire nouveau s'imposa au $\mathrm{XIX}^{\mathrm{e}}$ siècle, dans le cadre d'une compétition entre Argentan (ou Bayeux) et Alençon, où l'on utilisait une technique très différente (la couchure de fils) pour exécuter un champ de bride qui n'avait pas tout à fait le même aspect que l'ancien. Argentan revendiqua alors contre les altérations, dérivations, imitations voire contrefaçons de tout type, le savoir-faire authentique du champ de bride (renommé " grand réseau " par Lefébure), exécuté comme il l'était au XVIII siècle, et dont ses religieuses bénédictines étaient désormais les gardiennes. Les pièces, aux dessins de plus en plus sophistiqués, de " Point » toujours fait main, visaient à la prouesse et au spectaculaire. Elles haussèrent la dentelle à l'aiguille du niveau d'un produit de luxe, sensible aux modes, à celui d'une œuvre d'art pérenne, à conserver dans les musées. En cherchant à lutter contre la dentelle mécanique fabriquée au sein d'une manufacture concentrée qui vouait les ouvrières au salariat, il s'agissait de maintenir une industrie domestique considérée comme vertueuse et saine. La reprise de la production dentellière en milieu rural aurait aussi permis d'enrayer la " dépopulation des campagnes ». Citant Fernand Engerand, l'abbé Leboulanger, aumônier des bénédictines d'Argentan souhaitait que la

129. Voir par exemple Engerand, 1900 ; Leboulanger, 1908 et 1912 ; Moulinet, 1912 ; Poupet, 1913 ; puis Boulard, 1924, etc.

130. A contrario, Madame Despierres note la grande difficulté d'exécution de la bride par couchure : elle nécessite les compétences de deux ouvrières spécialisées : voir Despierres, 1886, p. 80. 
dentelle continuât à retenir les ouvrières « autour du clocher qui [avait] carillonné leur baptême ${ }^{131} »$.

\section{Sources}

Annuaire des cinq départements de l'ancienne Normandie, publié par l'Association normande, Hardel, Caen, 1834-.

Boulard Félix, La dentelle d'Alençon, ses origines, ses développements techniques, industriels, artistiques et historiques, Alençon, Imprimerie alençonnaise, 1924.

Bulletin de la Société historique et archéologique de l'Orne, Alençon, 1883-1951.

Celnart Élisabeth, Manuel des demoiselles ou Arts et métiers, qui leur conviennent et dont elles peuvent soccuper avec agrément, $1^{\text {re }}$ éd., Paris, Roret, 1826, p. 159163, $3^{e}$ éd. revue corrigée augmentée, 1828.

Chennevières Philippe DE, Notes d'un compilateur pour servir à l'histoire du Point de France, Lenoël-Hérouart, Amiens, 1869.

Cussy M. DE, «Rapport», Annuaire des cinq départements de l'ancienne Normandie, 1855.

Descriptions des arts et métiers, faites ou approuvées par Messieurs de l'Académie royale des sciences de Paris, Nouvelle édition publiée avec des observations, et augmentée de tout ce qui a été écrit de mieux sur ces matières, en Allemagne, en Angleterre, en Suisse, en Italie, 20 vol., Neuchâtel, Imprimerie de la Société typographique, 1771-1783, Calixte Volland, Paris, an VII [1798].

Despierres G[érasime], Histoire du Point d'Alençon depuis son origine jusqu'à nos jours, Renouard et H. Laurens, Paris et Alençon, 1886.

Encyclopédie méthodique ou par ordre des matières par une société de gens de lettres de savants et d'artistes. Commerce, t. 2, Panckoucke, Paris, Liège, Plomteux, 1783.

Engerand Fernand, "L'industrie de la dentelle en Normandie ", Revue des deux mondes, $\mathrm{n}^{\circ} 158$, mars-avril 1900, p. 645-664.

Jauber abbé Pierre et MacQuer Philippe, Dictionnaire raisonné universel des arts et métiers contenant l'histoire la description, la police des fabriques et manufactures de France et des pays étrangers, P. Fr. Didot, Paris, 1773.

Leboulanger Abbé J., Le Point et l'école dentellière d'Argentan, A. Mouville, Caen, 1925.

Leboulanger Abbé J., La dentelle d'Argentan, É. Langlois, Argentan, (1908), 1912.

Lefébure Ernest, Histoire de la dentelle à Bayeux de 1676 à 1900, J. Tueboeuf, Bayeux, 1913 (rééd. Conservatoire de la dentelle de Bayeux).

131. Leboulanger, 1912, p. 63. 
Lefrou M, "Rapport ", Annuaire des cinq départements de l'ancienne Normandie, 1858.

Jean Moulinet, La dentelle à l'aiguille en Basse-Normandie, É. Langlois, Argentan, 1912.

Macquer Philippe, Dictionnaire portatif des arts et métiers, Contenant en abrégé l'histoire, la description \& la police des arts et métiers, des fabriques et manufactures de France \& des pays étrangers, Lacombe, Paris, 1766.

Odolant-Desnos J., "De la fabrication du Point d'Alençon par la maison Docagne ", Journal d'Alençon, 10 juillet 1842, p. 1-2.

B.-J. Poupet, La dentelle d'Alençon, Thèse de doctorat, université de Paris, faculté de Droit, A. Rousseau, Paris, 1913.

Roland de la Platière Jean-Marie, Encyclopédie méthodique. Manufactures. Arts et métiers, Panckoucke, Paris, t. 1, 1785 et Recueil de planches de l'Encyclopédie par ordre de matières, t. 6, Panckoucke, Paris, 1786.

Diderot Denis et Le Rond D’Alembert Jean (dir.), Encyclopédie ou Dictionnaire raisonné des sciences, des arts et des métiers, t. 12, Paris, 1765 ; Supplément à l'Encyclopédie, Rey, Panckoucke et Robinet, Paris, 1776-1780.

Savary des Bruslons Jacques (éd.), Dictionnaire universel de Commerce, veuve Estienne, Paris, 1723, 1741.

\section{Bibliographie}

Audinet Isabelle, "La dentelle d'Argentan. Quand la tradition revit ", Patrimoine normand, février-mars 2000, extrait publié en ligne : http://www.patrimoine-normand.com/index-fiche-44379.html.

BufFÉvent Béatrix DE, L'économie dentellière en région parisienne au XVIT siècle, Société historique de Pontoise, Pontoise, 1984.

Capul Maurice, Les enfants placés sous l'Ancien Régime. 1. Abandon et marginalité; 2. Infirmité et hérésie, Privat, Toulouse, 1989 et 1990.

Clolus Nicolas, "La politique de répression et de conversion de l'intendant d'Alençon Lallemant de Lévignen envers les protestants (1726-1766) : une politique réussie ? ", Annales de Bretagne et des Pays de l'Ouest, n 113-2, 2006, p. 81-97.

Fouriscot Mick et STYCZYNsKi Christophe, L'histoire du Point d'Argentan, Didier Carpentier, Paris, 1997.

Garrigues Frédérique, "Les intendants du commerce au XVIII siècle ", Revue d'histoire moderne et contemporaine, Acteurs et pratiques du commerce dans l'Europe moderne, $\mathrm{n}^{\circ}$ 45/3, juillet-septembre 1998, p. 626-661.

KempF Gérard, "Recherches sur la dentelle ornaise ", Le Pays d'Argentan, n 84, 27 décembre 2010. 
Kempf Gérard, "Le "Poinct" de France à Argentan aux XVII et XVIII siècles ", Le Pays d'Argentan, $\mathrm{n}^{\circ}$ 31, septembre 1997 (numéro spécial).

LeNHof Jean-Louis, "Alençon en 1857 : apogée d'un modèle industriel et social ", Cahier des Annales de Normandie, n 35, 2009, p. 227-246.

Limon Béatrice, "Dentelle d'Argentan ou d'Alençon, ça change quoi ? ", OuestFrance, publié en ligne le 14 novembre 2015.

Mauboussin Johanna et Guillaumin Magali (dir.), Jolies ornaises. Dentelles jumelles d'Alençon et d'Argentan (Catalogue de l'exposition, Alençon, Musée des Beaux-arts et de la dentelle, 10 avril-6 novembre 2018, et Argentan, Maison des dentelles, 2 avril-2 novembre 2019), Alençon et Argentan, 2018.

Million Gérard, La dentelle d'Alençon, Recueil de textes, XVII -XX s., Archives départementales de l'Orne. Service éducatif, Conseil général de l'Orne, Alençon, 2001.

Minard Philippe, L'Inspection des manufactures en France, de Colbert à la Révolution, thèse de doctorat, université Paris I, 1994, 3 vols.

Musée des Beaux-Arts et de la dentelle (dir.), Le Point d'Alençon, Éditions de l'Étrave, Igé, 2011.

Plessix René (dir.), L’Orne de la préhistoire à nos jours, Éditions J.-M. Bordessoules, Saint-Jean-d'Angély, 1999.

Risselin-Steenebrugen Marie, Les dentelles italiennes aux Musées Royaux d'art et d'histoire, Musées royaux d'art et d'histoire, Bruxelles, 1973.

\section{Documents vidéo}

Une histoire de dentelle - Ville d'Argentan, Yolanda Communication, 2006.

Le savoir-faire de la dentelle au point d'Alençon, réalisé par Dino GAMBINI et produit par André Guéret, Atelier Cinéma Normandie, ACCAAN, 2003.

\section{L'autrice}

Brigitte Tambrun est chercheure au CNRS, au Laboratoire d'études sur les monothéismes (LEM, UMR 8584), université PSL; elle est membre du LabEx Hastec. 